\title{
An investigation into specialist practice nurses' knowledge of cardiopulmonary resuscitation guidelines in a tertiary hospital in Gauteng Province, South Africa
}

\author{
M L Botes, MSc; M Moepeng, MSc \\ Department of Nursing Education, Faculty of Health Sciences, University of the Witwatersrand, Johannesburg, South Africa
}

Corresponding author: M L Botes (Meghan.Botes@wits.ac.za)

\begin{abstract}
Background. Cardiac arrest is among the major causes of sudden deaths globally. Although out-of-hospital cardiac arrest occurs more commonly, in-hospital cardiac arrest is still a major health problem. Critical care areas provide care to critically ill patients who are at risk of cardiac arrest. It is important that nurses are knowledgeable and competent in cardiopulmonary resuscitation (CPR) in order to optimise the patient's chances of survival and quality of life after cardiac arrest.

Objective. To investigate specialist practice nurses' knowledge of evidence-based guidelines for CPR.

Methods. A descriptive cross-sectional survey was utilised. We sampled all critical care registered nurses ( $N=96)$ currently working in the adult emergency departments and intensive care units at Charlotte Maxeke Johannesburg Academic Hospital in Johannesburg, South Africa. A selfadministered instrument, the 'evaluation questionnaire on CPR knowledge for health personnel from emergency services' was used. Data were analysed using descriptive and comparative statistics.

Results. The mean CPR knowledge score was $46 \%$. A score of $84 \%$ was considered adequate for a pass, and no respondents achieved this score. The majority of the respondents $(80.85 \% ; n=76)$ were specialists in the field of intensive care nursing.

Conclusion. The CPR knowledge of specialist practice nurses was suboptimal for the care required in high-risk settings. Further training is indicated.

Keywords. specialist practice nurses; knowledge; cardiopulmonary resuscitation.

South Afr J Crit Care 2020;36(2):68-72. https://doi.org.10.7196/SAJCC.2020.v36i2.397
\end{abstract}

Contribution of the study. We showed that specialist nurses working in critical care environments at a public hospital in Johannesburg scored poorly in a CPR knowledge test.

Sudden cardiac arrest remains a leading cause of death, and continues to be an important public healthcare problem. ${ }^{[1]}$ In South Africa (SA), cardiovascular disease is the second leading cause of death after HIV/AIDS, ${ }^{[2]}$ responsible for one in six deaths. Since the introduction and recommendation of cardiopulmonary resuscitation (CPR) in the 1960s, it has become a widely used and standard treatment for the management of cardiac arrest. Although out-ofhospital cardiac arrest occurs more commonly, in-hospital cardiac arrest is still a major problem. A study that focused specifically on in-hospital cardiac care in developing countries found that of all the patients who had suffered cardiac arrest and received CPR, there was return of spontaneous circulation (ROSC) in only $7.4 \%(n=14)$ of the patients. ${ }^{[3]}$ Only $1.6 \%(n=3)$ of all the patients who suffered cardiac arrest survived for 24 hours. ${ }^{[3]}$ The results of this study are alarming, and low performance of CPR was identified as one of the contributing factors. It was suggested that ROSC rates can be influenced by the quality of hospital personnel training, adherence to evidence-based protocols and the implementation of internal quality-control mechanisms. ${ }^{[4]}$
In recent years, research on resuscitation has considerably increased. Guidelines for cardiac resuscitation have been implemented at an international level, and have undergone several substantial changes ${ }^{[3]}$ Prompt defibrillation of an individual who has suffered sudden cardiac arrest is the most important determinant of survival. ${ }^{[4]}$ Internationally, hospitals have worked to improve their in-hospital response to cardiac arrest, with the emphasis moving to post-cardiac arrest care. ${ }^{[5]}$

A study by Ocen et al. ${ }^{[3]}$ found that cardiac arrest occurred in $4.5 \%$ of patients in intensive care units (ICUs) within hospitals. These units and other critical care areas (which include emergency departments) provide care for critically ill patients with life-threatening conditions, requiring staff to have specialised knowledge and skills in life support to be able to save lives. Additional training in life support courses such as basic and advanced cardiac life support (BLS and ACLS) equip healthcare professionals with the most current knowledge and skills that are evidence-based and necessary for the management of life-threatening conditions such as cardiac arrest. It is vital that critical care nurses keep abreast with new developments in resuscitation science and technology. BLS includes CPR and emphasises minimal interruptions in chest 
compressions, providing compressions of adequate rate and depth, avoiding leaning between compressions, and avoiding excessive ventilation. ${ }^{[6]}$ Advanced level training in resuscitation is mostly aimed at healthcare professionals and skills learnt include rhythm recognition, defibrillation, cardioversion and airway management. ${ }^{[6]}$ Burkhardt et al. ${ }^{[7]}$ conducted a study to quantify the effect of knowledge on performance of all aspects of chest compressions, including rate, depth, recoil and hand positioning. They showed that respondents who identified effective compression rate to be $>100$ per minute performed chest compressions at a higher rate. On hand placement, respondents who knew proper hand placement performed a greater number of compressions. These findings suggested that knowledge of the guidelines had significant impact on CPR performance for at least some components of CPR.

In an in-hospital setting, nurses are often the first on the scene of cardiac arrest, initiating CPR as well as summoning assistance from the code team. It is therefore important that specialist nurses working in critical care areas are knowledgeable and competent in CPR to optimise the patient's chances of survival and quality of life. However, studies from across the globe report that nurses' knowledge of basic and advanced life support resuscitation guidelines are suboptimal. ${ }^{[1,20,21]} \mathrm{A}$ similar study conducted in Botswana confirmed that nurses' knowledge and skills related to CPR guidelines were poor. ${ }^{[8]}$

Studies have shown that training influenced knowledge and performance of CPR. ${ }^{[9]}$ According to Krajina et al. ${ }^{[9]}$ theoretical knowledge on how to perform CPR is essential for the ability to perform it in practice, and nurses with good theoretical knowledge achieve better CPR performance. In agreement, Roshana et al. ${ }^{[10]}$ showed that respondents who received some CPR training within 5 years obtained the highest mean score, and those who were involved in resuscitation frequently scored higher than those who were seldom or not involved in resuscitation. Certification on CPR should be renewed every 2 years. There is no legislative requirement for nurses to obtain BLS certification; however, healthcare establishments may choose to make it mandatory, and it is an assumed competency for a specialist practice nurse. ${ }^{[11]}$

In view of nurses' inadequate knowledge of CPR, as reported previously in $\mathrm{SA}^{\left[{ }^{[12]}\right.}$ the objective of this study was to investigate specialist practice nurses' knowledge of evidence-based American Heart Association (AHA) resuscitation guidelines in a universityaffiliated public sector hospital in Johannesburg, with an intention of making recommendations for clinical practice and education of critical care nurses. The secondary objectives included determining the difference in demographic characteristics and CPR knowledge, and the relationship between nurses' post basic specialisation as well as years of nursing experience and CPR knowledge

\section{Methods}

A descriptive cross-sectional survey was utilised to assess the knowledge of critical care nurses of CPR guidelines.

The setting for this study included two adult emergency departments (Trauma and Medical) and five ICUs, each unit with $7-12$ beds, at Charlotte Maxeke Johannesburg Academic Hospital, a public sector hospital in Johannesburg. The trauma and emergency department is a level I public sector trauma unit that accepts direct cases in need of highly specialised care. The ICUs include trauma, cardiothoracic, coronary care, neurosurgery and multidisciplinary or general ICU. These are highly specialised critical care settings, which accept critically ill patients from both medical and surgical disciplines.

\section{Population and sample}

Specialist practice emergency $(n=33)$ and intensive care $(n=63)$ nurses working in the hospital were eligible for participation in this study. A total of 94 participants were recruited to the study, and the response rate to the convenience sampling method was $97 \%$.

\section{Data collection}

A self-administered questionnaire was used. The 'evaluation questionnaire on CPR knowledge for health personnel from emergency services', derived from the recommendations of the 2010 AHA guidelines, updated for CPR and adapted for use in Spain by Garcia et al. ${ }^{[13]}$ was chosen as the most suitable instrument and was used with the permission of the authors. The tool was validated in a pilot study conducted by Garcia et al. ${ }^{[13]}$ For this study, face and content validity were ensured by an expert review panel of specialist nurses in the fields of emergency nursing, critical care and nursing education. An organised review of the instrument's contents was undertaken, and since the tool was used to measure knowledge of the 2010 AHA guidelines for $\mathrm{CPR}$ and cardiovascular care, some contents of the questionnaire were modified to include the 2015 updates, and then the tool was reviewed by a panel of experts to ensure face and content validity. Data were collected between November 2016 and February 2017.

The questionnaire comprised 20 questions on CPR, each with 4 possible responses and 1 correct answer. The questionnaire had 6 questions focused on BLS, and the rest of the questions were focused on ALS. Among the questions, 7 referred specifically to aspects updated in the 2010 clinical practice guideline on CPR for BLS and ALS.

\section{Data management and analyses}

Data were captured and cleaned using an Excel spreadsheet (Microsoft Corp., USA). Statistical analysis was performed using STATA software, version 10 (STATA Corp., USA). Respondents had to obtain a score of 17 out of $20(>85 \%)$ to be classified as having sufficient knowledge according to the 2010 AHA accreditation criteria.

Descriptive and comparative statistics were used to analyse the data. Percentage, mean and standard deviation were used to describe respondents' demographic data and nurses' theoretical knowledge. The significant difference between mean CPR knowledge score and age, gender, academic qualification, area of post-basic specialisation, years of experience and life support courses attended were established using Student's $t$-test. A $\chi^{2}$ test and linear regression models (univariate and multivariate) were computed to determine associations between specialist practice nurses' qualification as well as their years of experience and their knowledge of the CPR guidelines.

\section{Results}

Almost half of the respondents ( $46.8 \% ; n=44)$ were between the ages of 41 and 50 years, $82.98 \%(n=78)$ were female and $80.85 \%(n=76)$ were specialised in the field of intensive care nursing (Table 1).

The mean (standard deviation (SD) ) CPR knowledge score of the 94 respondents was $46 \%$ (12.71). This result indicated that none of the respondents passed the CPR knowledge test with a score above $85 \%$.

The percentage of nurses with $>5$ years, 5 - 10 years and $>10$ years of experience was $32.0 \%(n=30), 35.1 \%(n=33)$ and $33.0 \%(n=32)$, respectively.

The majority of the respondents $(80.9 \% ; n=76)$ had post-basic training in intensive care nursing, $95.7 \%(n=90)$ had trained in BLS and $46.8 \%(n=44)$ had been trained more than 2 years ago. 
We found no significant difference between CPR knowledge score and age, gender, academic qualification, area of post-basic specialisation, years of experience and life support courses attended by the respondents (Table 2). There was little variation in the mean CPR knowledge scores between the different age groups. The male respondents had a slightly higher mean CPR knowledge score (47.9\%) than the female respondents (45.77\%).

The type of academic qualification (degree or diploma) showed no variation in mean CPR score, as both groups averaged $46 \%$.

Although emergency care nurses had a slightly higher mean CPR score (48\%) compared with the intensive care nurses (45\%), both groups had suboptimal scores.

There was no significant difference in mean CPR score between respondents with $>10$ years of nursing experience $(58 \%)$ from nurseswith

\begin{tabular}{|c|c|}
\hline Characteristic & $n(\%)$ \\
\hline \multicolumn{2}{|l|}{ Age (years) } \\
\hline$<30$ years & $12(12.77)$ \\
\hline $31-40$ & $20(21.28)$ \\
\hline $41-50$ & $44(46.81)$ \\
\hline $51-60$ & 17 (18.09) \\
\hline$>61$ & $1(1.06)$ \\
\hline \multicolumn{2}{|l|}{ Gender } \\
\hline Female & $78(82.98)$ \\
\hline Male & $16(17.02)$ \\
\hline \multicolumn{2}{|l|}{ Qualification } \\
\hline Diploma & $74(78.72)$ \\
\hline Degree & $20(21.28)$ \\
\hline \multicolumn{2}{|l|}{ Post-basic specialisation } \\
\hline Trauma and emergency nursing & $18(19.15)$ \\
\hline Intensive care nursing & $76(80.85)$ \\
\hline \multicolumn{2}{|l|}{ Years of experience } \\
\hline$<5$ & $30(31.91)$ \\
\hline$>5-10$ & $33(35.11)$ \\
\hline$>10$ & $31(32.98)$ \\
\hline \multicolumn{2}{|l|}{ Attended life support courses } \\
\hline BLS & $90(95.74)$ \\
\hline ACLS & $20(21.28)$ \\
\hline ATLS & $12(12.77)$ \\
\hline PALS & $6(6.38)$ \\
\hline Other & $2(2.13)$ \\
\hline \multicolumn{2}{|l|}{ Last training in BLS } \\
\hline Within past 12 months & $21(22.34)$ \\
\hline 13 - 24 months & $25(26.6)$ \\
\hline$>24$ months & $44(46.81)$ \\
\hline \multicolumn{2}{|l|}{ Last training in ACLS } \\
\hline Within past 12 months & $2(2.13)$ \\
\hline $13-24$ months & $3(3.19)$ \\
\hline$>24$ months & $15(15.96)$ \\
\hline \multicolumn{2}{|l|}{ Last training in ATLS } \\
\hline Within past 12 months & $1(1.06)$ \\
\hline 13 - 24 months & $4(4.26)$ \\
\hline$>24$ months & $7(7.45)$ \\
\hline \multicolumn{2}{|l|}{ Last training in PALS } \\
\hline Within past 12 months & 0 \\
\hline 13 - 24 months & 0 \\
\hline$>24$ months & $6(6.38)$ \\
\hline
\end{tabular}

BLS = basic life support; ACLS = advance cardiac life support; ATLS = advanced trauma life support; PALS = paediatric advanced life support.
$<10$ years nursing experience (55\%). Similarly, no significant difference was found between intensive care nurses' and trauma and emergency nurses' scores in the CPR knowledge test using univariate analysis $(p=0451)$. This was also true for univariate analysis of scores between specialist nurses with $>10$ years' experience compared with those with $<10$ years' experience ( $p=0314$ ). Finally, we found no association between post-basic specialisation, years of nursing experience and the score in the CPR knowledge test using a multilinear regression model (Table 3).

\section{Discussion}

Respondents in this study represented a range of experience and ages for specialist practice nurses in a SA academic hospital. The majority had completed BLS courses, but not ALS courses. This may account in

Table 2. Mean CPR knowledge score by demographic characteristics

\begin{tabular}{|c|c|c|}
\hline Characteristic & Mean (SD) & $p$-value \\
\hline Age (years), one-way ANOVA & & 0.1535 \\
\hline$<30$ & $48.75(12.45)$ & \\
\hline $31-40$ & $49(13.04)$ & \\
\hline $41-50$ & $43.86(13.07)$ & \\
\hline $51-60$ & $44.71(10.23)$ & \\
\hline$>61$ & $70(0)$ & \\
\hline Gender ( $t$-test) & & 0.6866 \\
\hline Female & $45.77(12.54)$ & \\
\hline Male & $47.19(13.9)$ & \\
\hline Academic qualification ( $t$-test) & & 0.9967 \\
\hline Diploma & $46.01(12.66)$ & \\
\hline Degree & $46(13.24)$ & \\
\hline Area of post-basic specialisation ( $t$-test) & & 0.4508 \\
\hline Trauma and emergency nursing & $48.06(13.74)$ & \\
\hline Intensive care nursing & $45.53(12.51)$ & \\
\hline Years of experience (one-way ANOVA) & & 0.5204 \\
\hline$<5$ & $46(13.98)$ & \\
\hline$>5-10$ & $44.24(10.98)$ & \\
\hline$>10$ & $47.9(13.28)$ & \\
\hline Life support courses attended (one-way & & 0.8122 \\
\hline \multicolumn{3}{|l|}{ ANOVA) } \\
\hline BLS & $45.94(12.61)$ & \\
\hline ACLS & $49.5(13.56)$ & \\
\hline ATLS & 48.75 (11.89) & \\
\hline PALS & $43.33(6.06)$ & \\
\hline Other & $45(14.14)$ & \\
\hline $\begin{array}{l}\mathrm{CPR}=\text { cardiopulmonary resuscitation; BLS = basi } \\
\text { cardiac life support; ATLS = advanced trauma } \\
\text { life support; PALS = paediatric advanced life supp }\end{array}$ & support; ACLS = & \\
\hline
\end{tabular}

Table 3. Univariate and multivariate linear regression model illustrating the association between post-basic nursing specialisations, years of experience and scoring in the CPR knowledge test

\begin{tabular}{|c|c|c|c|c|}
\hline \multirow[b]{2}{*}{ Characteristic } & \multicolumn{2}{|c|}{ Univariate analysis } & \multicolumn{2}{|c|}{ Multivariate analysis } \\
\hline & $r(95 \% \mathrm{CI})$ & $p$-value & $r(95 \% \mathrm{CI})$ & $p$-value \\
\hline \multicolumn{5}{|c|}{ Area of post-basic specialisation } \\
\hline $\begin{array}{l}\text { Intensive care } \\
\text { nursing }\end{array}$ & $2.53(9.19-4.10)$ & 0.451 & $0.95(9.57-7.68)$ & 0.828 \\
\hline \multicolumn{5}{|c|}{ Years of experience } \\
\hline$>10$ & $2.82(2.71-8.36)$ & 0.314 & Omitted due to $\mathrm{cc}$ & -linearity \\
\hline
\end{tabular}


some respect for the suboptimal mean CPR knowledge score of $46 \%$ (12.71), because 14 of the 20 questions in the questionnaire are related to ALS. However, it is interesting to note that even respondents with ALS training obtained suboptimal scores.

The mean CPR score obtained in this study was higher than the $11 \%$ that was previously reported in a SA study that sampled all nurses and included questions only on BLS. ${ }^{[12]}$ While our results were similar to those reported for nurses in other developing countries including Botswana, ${ }^{[7]}$ where nurses obtained a mean CPR knowledge score of $55 \%$, and Bahrain, ${ }^{[14]}$ where nurses achieved a mean score of $42 \%$, these studies included all registered nurses. In Greece, ${ }^{[15]}$ nurses specialised in critical care obtained a mean score of $49.5 \%$. While knowledge retention is a major contributor to the inadequate scores, it is not known whether nurses are exposed to opportunities to refresh both knowledge and skills practically or educationally.

The scores for nurses in this study were better than those of doctors at a SA tertiary hospital who scored $41 \%$ for adult CPR and $37 \%$ for paediatric CPR. ${ }^{[16]}$ Moreover, a study to determine competency of CPR among intermediate qualified SA emergency medical service personnel found that the median knowledge score was $50 \%$ and median skill score was $33 \%$, indicating knowledge and skill performance in CPR below standard in critical care health personnel. ${ }^{[17]}$ Responders to patients suffering sudden cardiac arrest include various members of the multidisciplinary team, and therefore all members should be required to stay updated.

Nurses' inadequate theoretical knowledge of CPR has been attributed to ineffective initial or refresher training. Instructor competence, teaching methods, poor recall of knowledge and infrequency of updates are other reasons that influence poor CPR knowledge among nurses. Bukiran et al. ${ }^{[18]}$ and Marzooq and Lyneham ${ }^{[14]}$ attributed suboptimal CPR knowledge to the lack of motivation to update knowledge, which was reported by $1.7 \%$ of their respondents, while $14 \%$ blamed lack of institutional guidance.

Nearly half of our respondents had expired BLS certificates, while two-thirds had not renewed their ALS certificates for more than 24 months. This illustrates that nurses are not updating or renewing their certification of life support courses. The percentage of respondents who had renewed their BLS certificate in the last 12 months in our study was higher than the $1.3 \%(n=1)$ reported by Passali et al. ${ }^{[14]}$ in Greece, where the mean time elapsed since last BLS course training was 15 years. Often, it is an expectation of nurses working in a public facility that training be provided for them by the institution. However, there is an apparent lack of intrinsic motivation for self-development, and responsibility is placed on management of the health institution.

Previous studies have shown that knowledge of the CPR guidelines was significantly associated with academic qualification. ${ }^{[19,14]}$ In fact, a study conducted by Al-Janabi and $\mathrm{Al}-\mathrm{Ani}^{[20]}$ found that $74.3 \%$ of nurses with degree qualifications scored above the cut-off point on CPR knowledge in Baghdad, compared with $22.9 \%$ of those with a diploma. We did not find a significant relationship between type of qualification and mean CPR scores. This may be due to the fact that undergraduate and post-basic nursing education do not include BLS, and therefore do not affect this specific knowledge after qualification.

Although there was no significant difference between demographic characteristics and CPR knowledge when comparing the trauma and emergency nurses and intensive care trained nurses, the former appear to have a slightly higher mean score (48\%) compared with the latter (45\%), and nurses with $>10$ years of nursing experience scored slightly higher (58\%) than nurses with $<10$ years $(55 \%)$. These results differ from those of Parajulee and Selvaraj, ${ }^{[21]}$ who reported significant associations between CPR knowledge, years of working experience and work area. Their study included nurses working in a teaching hospital, so their results may not be comparable to our findings.

\section{Study limitations and recommendations}

Studies on CPR knowledge and skill retention report a decline in knowledge level over time, with poor performance $3-6$ months post training. Hence it is important for nurses to undergo frequent training on CPR knowledge to keep up to date with all updates from the AHA. This requirement can be incentivised through the continuous professional development (CPD) points system, where BLS and ALS training can form part of recognised CPD programmes. The AHA certificate courses including BLS are valid for 2 years, and it is recommended that specialist practice nurses renew their certificates every 2 years.

CPD plans for nurses should include mandatory updates of vital knowledge and skills such as those included in the BLS course.

Although the literature suggests that a relationship exists between knowledge and practice, the focus of this study was limited to the area of knowledge, and therefore may not accurately reflect actual practice or skill in performing CPR. Another limitation of this study is that the data were collected from only one institution. Face and content validity of the instrument were ensured by an expert review panel with the assistance of the original authors; however, the reliability coefficient of the instrument was not reported by the original authors.

\section{Conclusion}

Specialist nurses scored poorly on the CPR knowledge test, indicating their suboptimal knowledge. Therefore, we recommend that nurses undergo frequent training on CPR knowledge to keep up to date with all the updates from AHA.

Declaration. This study was conducted in partial fulfilment of a Master of Science degree in Nursing.

\section{Acknowledgements. None.}

Author contributions. MM designed the study and analysed the data under the supervision of MLB. MLB wrote the article. Both authors approved the article for publication.

Funding. None.

Conflicts of interest. None.

1. Wong CX, Brown A, Lau DH, et al. Epidemiology of sudden cardiac death: Global and regional perspectives. Heart Lung Circ 2019;28(1):6-14. https://doi.org/10.1016/j.hlc.2018.08.026

2. Nojilana B, Bradshaw D, Pillay-van Wyk V, et al. Persistent burden from non-communicable diseases in South Africa needs strong action. S Afr Med J 2016;106(5):23-24. https://doi. org/10.7196/samj.2016.v106i5.10776

3. Ocen D, Kalungi S, Ejoku J, et al. Prevalence, outcomes and factors associated with adult in-hospital cardiac arrests in a low-income country tertiary hospital: A prospective observational study. BMC Emerg Med 2015;15:23. https://doi.org/10.1186\%2Fs12873-015-0047-0

4. McCoy M, Schultz A, Graham R. Strategies to improve cardiac arrest survival: A time to act. Washington (DC): National Academies Press (US); 2015. https://www.ncbi.nlm.nih.gov/books/ NBK321499/ (accessed 3 May 2019).

5. Lai C-Y, Lin F-H, Chu H, et al. Survival factors of hospitalised out-of-hospital cardiac arrest patients in Taiwan: A retrospective study. PloS ONE 2018;13(2):e0191954. https://doi. org/10.1371/journal.pone.0191954

6. Meaney PA, Sutton RM, Tsima B, et al. Training hospital providers in basic CPR skills in Botswana: Acquisition, retention and impact of novel training techniques. Resuscitation 2012;83(12):1484-1490. https://doi.org/10.1016\%2Fj.resuscitation.2012.04.014

7. Burkhardt JN, Glick JE, Terndrup TE. Effect of prior cardiopulmonary resuscitation knowledge on compression performance by hospital providers. West J Emerg Med 2014;15(4):404-408. https://doi.org/10.5811/westjem.2014.1.19636

8. Rajeswaran L, Cox M, Moeng S, Tsima BM. Assessment of nurses' cardiopulmonary resuscitation knowledge and skills within three district hospitals in Botswana. Afr J Prim Health Care Fam Med 2018;10(1):e1-6. https://doi.org/10.4102/phcfm.v10i1.1633 
9. Krajina I, Kvolik S, Steiner R, Kovacevic K, Lovric I. Cardiopulmonary resuscitation, chest compression only and teamwork from the perspective of medical doctors, surgeons and anaesthesiologists. Iran Red Crescent Med I 2015;17(3):e18208. https://doi.org/10.5812/ ircmj.18208

10. Roshana S, Batajoo KH, Piryani RM, Sharma MW. Basic life support: Knowledge and attitude of medical/paramedical professionals. World J Emerg Med 2012;3(2):141-145. https://doi.org/10.58 47\%2Fwjem.j.issn.1920-8642.2012.02.011

11. South African Nursing Council. Competencies for critical care nurse specialist. SANC, March 2014

12. Keenan M, Lamacraft G, Joubert G. A survey of nurses' basic life support knowledge and training at a tertiary hospital. Afr J Health Prof Edu 2009;1(1):3. https://www.ajhpe.org.za/index.php/ ajhpe/article/view/15 (accessed 16 August 2020).

13. García S, Belen A, Alemán F, et al. Assessment of the knowledge level and its relevance in term of CPR in medical personnel of the hospital emergency medical system of the autonomous community of the region of Murcia. EnfermerÃa Glob 2015;14(39):246.

14. Marzooq H, Lyneham J. Cardiopulmonary resuscitation knowledge among nurses working in Bahrain. Int J Nurs Pract 2009;15(4):294-302. https://doi.org/10.1111/j.1440-172x.2009.01752.x

15. Assali C, Pantazopoulos I, Dontas I, et al. Evaluation of nurses' and doctors' knowledge of basic \& advanced life support resuscitation guidelines. Nurse Edu Pract 2011;11(6):365-369. https://doi. org/10.1016/j.nepr.2011.03.010
16. Botha L, Geyser M, Engelbrecht A. Knowledge of cardiopulmonary resuscitation of clinicians at a South African tertiary hospital. S Afr Fam Prac 2012;54(5):447-454. https://doi.org/10.1080/2 0786204.2012.10874269

17. Veronese J-P, Wallis L, Allgaier R, Botha R. Cardiopulmonary resuscitation by emergency medical services in South Africa: Barriers to achieving high-quality performance. Afr J Emerg Med Rev Afr Med Urgence 2018;8(1):6-11. https://doi.org/10.1016/j.afjem.2017.08.005

18. Bukiran A, Erdur B, Ozen M, Bozkurt AI. Retention of nurses' knowledge after basic life support and advanced cardiac life support training at immediate, 6-month, and 12-month post-trainin intervals: A longitudinal study of nurses in Turkey. J Emerg Nurs 2014;40(2):146-152. https://do org/10.1016/j.jen.2012.08.011

19. Elazazay $\mathrm{H}$, Abdelazez A, Elsaie O. Effect of cardiopulmonary resuscitation training program on nurses knowledge and practice. Life Sci J 2012;9(4):3494-3503. https://doi.org.10.1.1.381.3517

20. Al-Ani BAJ, Al-Janabi MA al-KM. Assessment of nurses' knowledge towards cardiopulmonary resuscitation at Al-Najaf City's teaching hospital. Kufa J Nurs Sci 2014;4(1):208-217.

21. Paralujee S, Selvaraj V. Knowledge of nurses towards cardio-pulmonary resuscitation in a tertiary care teaching hospital in Nepal. J Clin Diagn Res 2011;5(8):1585-1588.

Accepted 2 July 2020. 